\title{
Clinical Consequences of Untreated Dental Caries Evaluated with the Pulpal Involvement- Roots-Sepsis Index in the Primary Dentition of School Children from the Raipur and Durg Districts, Chhattisgarh State, India
}

\author{
Sansriti Tiwari Alok Dubey Bimaldeep Singh Alok Avinash \\ Department of Pedodontics and Preventive Dentistry, Rungta College of Dental Sciences and Research, Bhilai, India
}

\section{Key Words}

Primary dentition · Dental caries · PRS index

\begin{abstract}
Objective: To evaluate the prevalence of the clinical consequences of untreated dental caries in primary dentition among 6- to 8-year-old children using the prs [pulpal involvement ( $p)$, roots $(r)$ and sepsis (s)] index and record the distribution of prs among boys and girls. Subjects and Methods: The study included a sample of 371 children aged 6-8 years with at least one carious primary molar tooth, who had been randomly selected from schools in the districts of Raipur and Durg, Chhattisgarh State, India. We recorded the presence of decayed, extracted and filled (i.e. def) teeth. The prevalence of the consequences of untreated dental caries was evaluated using the prs index. The data were statistically analyzed to express the prevalence of prs among boys and girls and the distribution of individual prs codes in the sample population. Results: The prevalence of carious lesions was very high (87\%) and teeth with clinical consequences of untreated dental caries were found in $49.3 \%$ of the children. The prs distribution in children who were 8 years old (40.9\%) was higher than in the 6-year-old children (25.6\%). In the group of 8-year-olds, the occurrence of prs was greater among the boys whereas in the 6- and 7-yearold group, it was greater among the girls. Conclusion: The
\end{abstract}

prs index is a valuable tool for measuring the clinical consequences of untreated dental caries. It could be useful for epidemiological studies and provide relevant information regarding treatment needs.

(c) 2014 S. Karger AG, Basel

\section{Introduction}

Oral health conditions in high-income countries have improved. However, dental caries continues to be a major public health problem [1], especially in low- and middleincome countries [2-4]. Dental decay is very common in childhood [5], and if left untreated, the disease advances, with a significant impact on the child's general health, development, quality of life, productivity, school attendance and educational performance, maybe even resulting in hospitalization [6-8].

Public costs for the treatment of severe caries are obviously higher than for early carious lesions. Assessing the severity of caries is therefore an essential component of epidemiological surveys [9]. The dmft (decayed, missing and filled teeth) index has been considered a classic index for the measurement of carious process for the last 70 years, but it does not diagnose all the caries stages [10-12] or provide any information regarding the consequences of untreated caries (which may be more serious than ear-

\begin{tabular}{ll}
\hline KARGER 125:s & $\begin{array}{l}\text { () 2014 S. Karger AG, Basel } \\
1011-7571 / 14 / 0242-0184 \$ 39.50 / 0 \quad \text { Karger }\end{array}$ \\
$\begin{array}{l}\text { E-Mail karger@karger.com } \\
\text { www.karger.com/mpp }\end{array}$ & $\begin{array}{l}\text { This is an Open Access article licensed under the terms of the } \\
\text { Creative Commons Attribution-NonCommercial 3.0 Un- } \\
\text { ported license (CC BY-NC) (www.karger.com/OA-license), } \\
\text { applicable to the online version of the article only. Distribu- } \\
\text { tion permitted for non-commercial purposes only. }\end{array}$
\end{tabular}

Dr. Sansriti Tiwari

Department of Pedodontics and Preventive Dentistry

Rungta College of Dental Sciences and Research

Kohka-Kurud Road, Bhilai 490024 (India)

E-Maildrsansriti@gmail.com 
ly caries) [13]. In 2007, in view of the growing burden of oral disease worldwide, the WHO emphasized the enhancement of action based on comprehensive data collection systems [14]. In 2010, realizing the urgent need to establish a scoring system for the advanced stages of caries, Monse et al. [13] introduced an index of the clinical consequences of untreated dental caries and named it the PUFA/pufa index (lowercase letters are used for primary dentition analysis). This index measures pulpal involvement $(\mathrm{P})$, ulceration of soft tissues $(\mathrm{U})$, fistula $(\mathrm{F})$ and abscess (A). It represented a breakthrough for the estimation of advanced carious lesions and it has been a successful tool ever since.

The PUFA index does not, however, present the treatment needs, which are an essential goal of any epidemiological index [15], as it can assist health-care planners and policy makers in the planning and provision of effective services. Another disadvantage of the PUFA/pufa index is that the code ' $\mathrm{P}$ ' includes two different clinical situations in a single code, i.e. (1) a tooth with a carious process extending to the pulp that could be endodontically treated and preserved and (2) a tooth with extensive caries that results in a grossly decayed crown with only the roots remaining, which may be indicated for extraction [15]. The need for the ulceration code appears superfluous, and many research studies have stated that it is the most rarely diagnosed PUFA code $[6,9]$. There is also an unnecessary extension of the index by including fistula and abscess as separate entities because these are the clinical presentation of the same condition and may interchange $[10,16]$.

Taking into considerations the doubts regarding the PUFA index, in 2013, Bagińska and Stokowska [15] introduced a new method for describing the clinical consequences of untreated dental caries and named it the PRS/ prs index. It measures pulpal involvement $(\mathrm{P})$, roots $(\mathrm{R})$ and sepsis (S), with each code representing a specific clinical manifestation and the most appropriate treatment needs. The aim of this study was to evaluate the status of primary dentition using the def (decayed, extracted and filled) index and to assess the clinical consequences of untreated dental caries using the prs index among children aged 6-8 years.

\section{Subjects and Methods}

This study was conducted in the districts of Raipur and Durg, Chhattisgarh State, India. Approval for the study was obtained from the Institutional Review Board of the Rungta College of Dental Sciences and Research. The study was explained to the heads of the institutions of the 7 schools selected and their permission was obtained prior to the commencement of the study. The students were given written consent forms 3 days before the study and only those returning with a signed form from their parents or guardians were included.

The study was preceded by a calibration session for the investigator comprising theoretical and practical sessions. The theoretical training was based on the PUFA index [13] and the PRS index [15]. The practical part consisted of B.S. training the investigator on 10 school children. Calibration of the examiner was done on 20 school children who were examined twice using the diagnostic criteria on successive days; the results were then compared to ascertain the diagnostic variability.

A pilot study was conducted on 150 school children aged 6-8 years. The prevalence of dental caries was calculated to determine the sample size for the main study. Four hundred and twenty-six children aged 6-8 years who were randomly selected from schools in the districts of Raipur and Durg were invited to participate. Children with at least one carious primary molar tooth were included in the study. The exclusion criteria were a lack of approval by a school to conduct the study and a child's absence from school on the day of the examination. The study was conducted on 371 children: 6-year-olds ( $\mathrm{n}=95,39$ boys and 56 girls), 7-year-olds $(\mathrm{n}=124,62$ boys and 62 girls) and 8-year-olds $(\mathrm{n}=152,84$ boys and 68 girls). The total sample included 185 boys and 186 girls. The oral examination was conducted under artificial light using a dental mirror. A ball-ended dental probe was used for the assessment of dental status by def index [17] as proposed by Grubell [18]. In accordance with the PUFA recommendations, no dental probe was used for the scoring of the prs index [13]. All children were instructed to brush their teeth before the examination.

The clinical consequences of untreated dental caries were assessed using the prs index [15] according to the following diagnostic criteria. Teeth with a carious process extending to the pulp which could be properly restored after an endodontic treatment were coded as 'p', i.e. pulpal involvement. Teeth with a grossly decayed crown with only roots remaining in the oral cavity and with a poor prognosis for direct/indirect restorative treatment were indicated for extraction and were coded as ' $r$ ', i.e. roots. Teeth with a pus-releasing sinus tract or a pus-containing swelling related to the tooth were indicated for extraction and were coded as 's', i.e. sepsis.

The mean def was calculated to express the presence of caries. The clinical consequences of untreated dental caries were assessed by calculating the prevalence and mean prs. The data obtained were statistically analyzed using SPSS software v16. The differences in the layout of prs components among the boys and the girls of each age group were analyzed and $p<0.05$ was considered statistically significant. An unpaired Student $t$ test was used for comparison between boys and girls in the different age groups. The kappa coefficient was used to determine intraexaminer reliability.

\section{Results}

Agreement for the assessment was $88 \%$. Of the 426 children invited to participate in the study, 371 had a def $>0$, indicating a high prevalence $(87 \%)$ of def in the surveyed population. Of these 371 children, severe caries (i.e. 


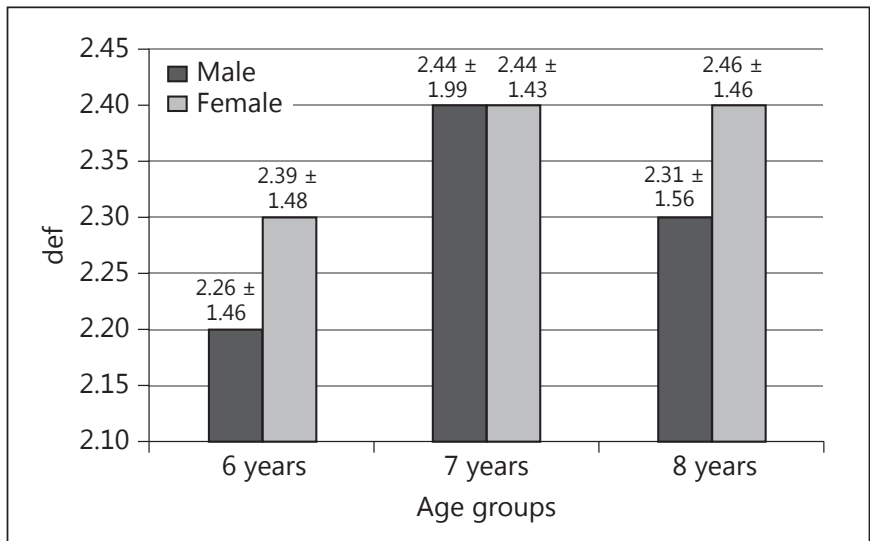

Fig. 1. Mean def in the boys and girls of the three age groups.

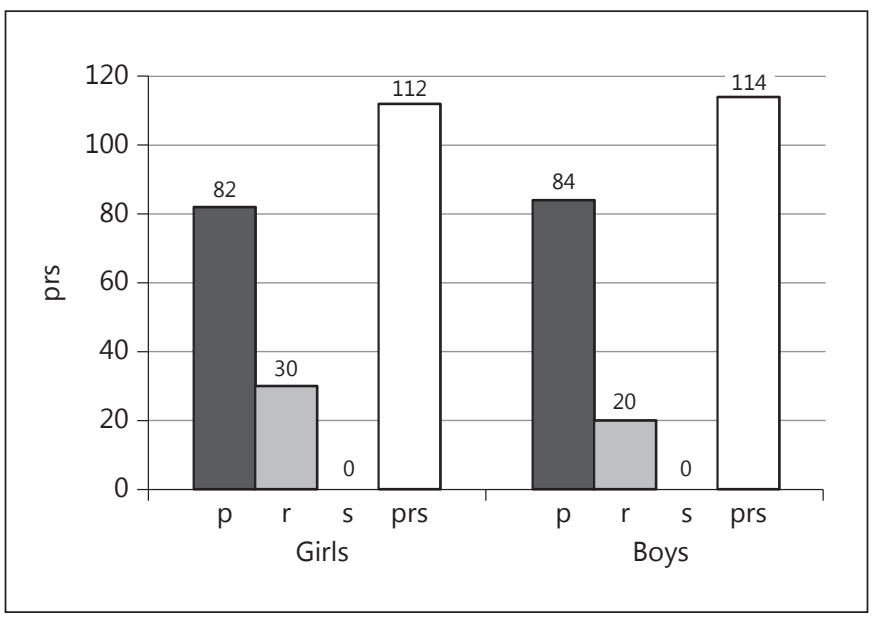

Fig. 2. Frequency distribution of prs and its individual codes $(\mathrm{p}, \mathrm{r}$ and $\mathrm{s})$ in the boys and girls.

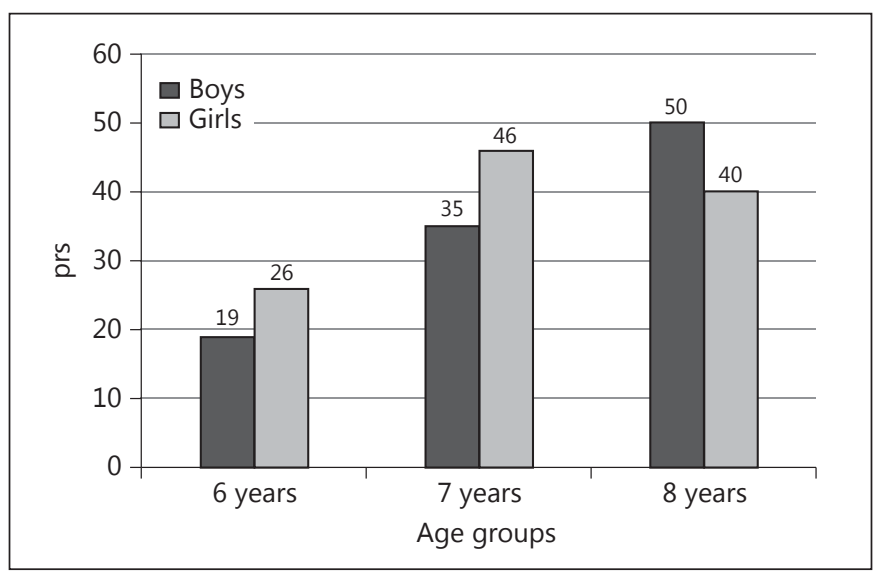

Fig. 3. Frequency distribution of prs in the boys and girls of the three age groups. prs $>0$ ) was observed in 183 (49.3\%), and 188 (50.7\%) had untreated caries without any clinical symptoms of pulpal or apical inflammation. The kappa coefficient for prs was estimated at 0.88, which represents a good level of intraexaminer reliability. The mean def index values recorded for the boys and girls of each age group are presented in figure 1 . The mean def value among boys and girls in the group of 6-year-olds was $2.26 \pm 1.46$ and 2.39 \pm 1.49 , respectively. In the 7 -year-old group, it was $2.44 \pm$ 1.99 and $2.44 \pm 1.43$, respectively. In the 8-year-old group, it was $2.31 \pm 1.56$ and $2.46 \pm 1.46$, respectively. Only 5 teeth were coded as 'e' (extracted) and only 12 teeth with an 'f score (filled) were observed.

The prevalence of prs and its individual codes was higher among boys than girls (fig. 2). When the different age groups were considered individually, this prevalence was higher in the 8-year-old children (40.9\%) than in the 6 -year-old group (25.6\%). The girls had higher prs scores in the 6- and 7-year-old group whereas in the 8-year-old group, the score was higher among the boys (fig. 3). Pulpal involvement was seen in 166 teeth $(44.7 \% ; \mathrm{p}=0.077)$. Root involvement was seen in 50 teeth $(13.4 \%)$ and $\mathrm{p}=$ 0.006 was statistically significant. No child with sepsis was observed.

\section{Discussion}

Our study population was characterized by a very high prevalence of dental caries, with $87 \%$ having a def score $>0$. The scores obtained for teeth extracted and filled due to caries was very low; this reflects negligence in the treatment of deciduous teeth. High levels of dental caries are probably due to the high consumption of cariogenic food, improper oral health practices, a lack of regular dental checkups, poor awareness and a lack of dental services available to children. The prevalence that we observed was higher than that reported by Joshi et al. [19] (77\%) and Grewal et al. [20] (67\%) among Indian children in the Tamil Nadu State and the Nainital district, respectively.

As children grow, there naturally is a continuous loss of deciduous teeth as a result of the physiological shedding of teeth. Inspite of this, we observed that the severity of dental caries in the deciduous teeth increased markedly along with the age of the children. Similar data were reported using the pufa index in 5- to 7-year-olds in the Bialystok district in Poland [21] and in a study by Shankar et al. [22] using the def index in a group of Indian children. The results clearly show that the neglect of caries treatment at an early stage increases the risk and severity 
of its clinical consequences over time. This necessitates an effective treatment planning for children and hence the need for an epidemiological index that could present the treatment modalities in the most appropriate manner.

In developed countries, the percentage of deciduous teeth undergoing treatment is high, reaching as much as $84 \%$ [23], but such treatment is commonly neglected in developing countries. Our study revealed a high negligence of treatment, with only 12 teeth scored under the 'filled' component of the def index, despite the high prevalence of caries (87\%) in the sample population. Several epidemiological studies in the literature have revealed a worse state of dentition in rural areas [24].

Limited data are available in the literature regarding the severity and sequelae of the carious process [9]. PUFA and PRS are the proposed indices for the assessment of the clinical consequences of untreated dental caries, but only the PRS index expresses the treatment needs. Bagińska and Stokowska [15] evaluated the prs index in Polish children aged 6-8 years and revealed that $40 \%$ of them had a prs score of $>0$; this is lower than in our study. The other data available in the literature concerning the assessment of the clinical consequences of untreated dental caries were obtained with the PUFA/pufa index. In our study, the prevalence of the clinical consequences of untreated dental caries was found to be similar to that in Polish children (43.4\%) [15]. It was reported to be much higher in Filipino children (84\%) [13] and lower in Brazilian children (23.7\%) [6].

We chose to use the prs index rather than the pufa index because the former not only presents the prevalence and experience of the advanced stages of caries but also provides health-care planners and policy makers with clear and relevant information regarding the treatment needs of the children with advanced caries. Moreover, the presentation of the components of prs presents the clinical consequences of untreated caries in a simple and effective manner. Unlike the pufa index, the prs index does not overestimate the occurrence of pulpal involvement. In the study by Bagińska and Stokowska [15], 184 molars diagnosed as pulpal involvement according to the pufa index were coded as roots according to the prs index and were indicated for extraction. In the prs index, the ulceration code has been eliminated, with teeth being coded as either pulpal involvement or roots as dictated by the clinical presentation of the etiology. In addition, fistula and abscess are included as a single code 'sepsis', thereby avoiding an unnecessary extension of the index [15].

\section{Conclusion}

The population evaluated in this study revealed that there is negligence in the dental treatment of children. There was a high prevalence of severe caries among the Indian children. The prs index is a valuable measurement tool for evaluating the clinical consequences of untreated dental caries and has an appropriate presentation of treatment needs. It could be considered as an alternative to the PUFA index. Further studies covering larger sample populations should be planned using the prs index.

\section{References}

1 Petersen PE: The World Oral Health Report. Continuous improvement of oral health in the 21 st century - the approach of the WHO Global Oral Health Programme. Community Dent Oral Epidemiol 2003;31:3-24.

2 Ajayi YO, Sofola OO: Descriptors of permanent teeth with cariously exposed pulp in patients presenting at a Nigerian hospital. Acta Odontol Scand 2013;71:1348-1350.

3 Mobarak EH, Shabayek MM, Mulder J, et al: Caries experience of Egyptian adolescents: does the atraumatic restorative treatment approach offer a solution? Med Princ Pract 2011;20:545-549.

4 Jafarian M, Etebarian A: Reasons for extraction of permanent teeth in general dental practices in Teheran, Iran. Med Princ Pract 2013;22:239-244.
5 Edelstein BL: The dental caries pandemic and disparities problem. BMC Oral Health 2006; 6:S2.

6 Figueiredo MJ, de Amorim RG, Leal SC, et al: Prevalence and severity of clinical consequences of untreated dentine carious lesions in children from a deprived area of Brazil. Caries Res 2011;45:435-442.

7 Leal SC, Bronkhorst EM, Fan M, et al: Untreated cavitated dentine lesions: impact on children's quality of life. Caries Res 2012;46: 102-106.

8 Benzian H, Monse B, Heinrich-Weltzien R, et al: Untreated severe dental decay: a neglected determinant of low body mass index in 12-year-old Filipino children. BMC Public Health 2011;11:558.
9 Bagińska J, Rodakowska E, WilczyńskaBorawska $\mathrm{M}$, et al: Index of clinical consequences of untreated dental caries (PUFA) in primary dentition of children from north-east Poland. Adv Med Sci 2013;58:442-447.

10 Frencken JE, de Amorim RG, Faber J, et al The Caries Assessment Spectrum and Treatment (CAST) index: rationale and development. Int Dent J 2011;61:117-123.

11 Ismail AI: Visual and visuo-tactile detection of dental caries. J Dent Res 2004;83:C56-C66.

12 Honkala E, Runnel R, Honkala S, et al: Measuring dental caries in the mixed dentition by ICDAS. Int J Dent 2011;2011:150424.

13 Monse B, Heinrich-Weltzien R, Benzian H, et al: PUFA - an index of clinical consequences of untreated dental caries. Community Dent Oral Epidemiol 2010;38:77-82.
Clinical Consequences of Untreated Dental Caries Using the prs Index
Med Princ Pract 2015;24:184-188 
14 World Health Organization: Oral health: action plan for the promotion and integrated disease prevention. Agenda item 17, 60th World Health Assembly, September 2007.

15 Bagińska J, Stokowska W: Pulpal involvement-roots-sepsis index: a new method for describing the clinical consequences of untreated dental caries. Med Princ Pract 2013; 22:555-560.

16 De Souza AL, van der Sanden WJM, Leal SC, et al: The Caries Assessment Spectrum and Treatment (CAST) index: face and content validation. Int Dent J 2012;62:270-276.

17 World Health Organization: Oral Health Surveys. Basic Methods, ed 4. Geneva, World Health Organization, 1997, chapt 5: Assessment form, pp 21-52.
18 Soben P: Essentials of Preventive and Community Dentistry, ed 4. New Delhi, Arya (Medi) Publishing House, 2009, pp 499-505.

19 Joshi N, Rajesh R, Sunitha M: Prevalence of dental caries among school children in $\mathrm{Ku}$ lasekharam village: a correlated prevalence survey. J Indian Soc Pedod Prev Dent 2005;23: 138-140.

20 Grewal H, Verma M, Kumar A: Prevalence of dental caries and treatment needs in the rural child population of Nainital District, Uttaranchal. J Indian Soc Pedod Prev Dent 2009; 27:224-226.

21 Bagińska J: Evaluation of the status of primary dentition in 6-7-year-old children from Bialystok District using the Mean DMF and the Index of Clinical Consequences of Untreated Caries (PUFA). Dent Med Probl 2013;50: 160-166.
22 Shankar S, Naveen N, Kruthika M, et al: Comparison of def index with Nyvad's new caries diagnostic criteria among three- to six-yearold children in a school at Bangalore city. Indian J Dent Res 2012;23:135-139.

23 Tickle M, Milsom K, King D, et al: The fate of the carious primary teeth of children who regularly attend the general dental service. $\mathrm{Br}$ Dent J 2002;192:219-223.

24 Bagińska J, Wilczyńska-Borawska M, Stokowska W: Incidence and intensity of dental caries in zero class schoolchildren in small towns of Podlaskievoivodeship. Magazyn Stomatol 2006;16:56-58. 